\title{
A Full-Range of 3D Body Scanning Solutions
}

\author{
Jean-Loup RENNESSON \\ TELMAT Industrie SA, Soultz, France
}

\begin{abstract}
TELMAT extends its range of 3D body scanning solutions named SYMCAD ${ }^{\mathrm{TM}}$ to fulfill a wide scope of applications in the apparel and fashion industry (retail shops, tailor-made clothing shops, online catalog shopping, body size measurement surveys) as well as in ergonomics (anthropometric studies), sport, health and fitness, entertainment, etc.

An overview of the miscellaneous technologies used by the new range of SYMCAD ${ }^{\mathrm{TM}}$ body scanners is given with an assessment of their adequacy for the considered applications. This analysis includes the following topics: time of scanning, calibration, 3D resolution, automatic detection of anatomical landmarks, automatic body measurement extraction, special tracking of active landmarks set on the subject's body, creation of 3D avatars, ...
\end{abstract}

Keywords: 3D body scanning technologies, white-light scanning, Kinect ${ }^{\circledR}$ or $\mathrm{Xtion}^{\circledR}$ depth sensor, automated body measurement, made-to-measure, mass customization

\section{Introduction}

TELMAT Industrie is a manufacturer of 3D body scanning solutions, based on white light projection, more precisely on the combination of projection of highly-encoded structured (white) light and stereovision.

Its range of 3D body scanning systems, named SYMCAD, is commonly used for the issue of military uniforms or corporate apparel (SYMCAD ${ }^{\text {TM }}$ Army solution). These 3D body scanning systems have also been deployed in the fashion industry, notably in tailor-made clothing shops, thanks to their ease-of-use, consistency and reliability. TELMAT now extends this range of 3D body scanning solutions named SYMCAD ${ }^{\mathrm{TM}}$ to fulfill an even wider scope of applications in the apparel and fashion industry (tailor-made clothing shops, retail shops, online catalog shopping, body size measurement surveys) as well as in ergonomics (anthropometric studies), sport, health and fitness, entertainment, etc.

\section{A wide scope of applications}

\subsection{Tailor-made clothing shops}

For tailor-made clothing shops, TELMAT developed the special SYMCAD ${ }^{\text {TM }}$ Tailor solution which is based on the SYMCAD ${ }^{\mathrm{TM}}$ II technology, a combination of the projection of highly-encoded structured light and optical triangulations between sets of two high resolution cameras (stereo vision) - see figure 1.

Firstly, the use of an appropriate encoding of the structured light, known as Gray code (see figure 2), improves the numeration of stripes and therefore leads to a much more robust 3D reconstruction, i.e. it avoids problems encountered by single-pattern projection devices, such as gaps between parts of the reconstructed 3D surfaces, or even sometimes, holes in the 3D dataset. Indeed, when two stripes deformed on the body are close to each other or when stripe colour confuses with underwear or skin colours, they merge at certain locations and it leads to a wrong numeration of stripes and the failure of the 3D-reconstruction.

Secondly, the projection of additional patterns, based on the Phase Shifting method (see figure 3) enables a pixel-wise 3D reconstruction and highly improves the resolution of the 3D dataset.

This SYMCAD ${ }^{\mathrm{TM}}$ II technology is ultra-fast and accurate. It takes less than a minute for the equipment to start up. The cycle includes an automatic scope calibration for measurement, totally free of any human intervention, with an acquisition time of 0.5 seconds for the entire human body and a $3 \mathrm{D}$ resolution higher than 25 points per sq. $\mathrm{cm}$., SYMCAD II is unequalled on the market. 


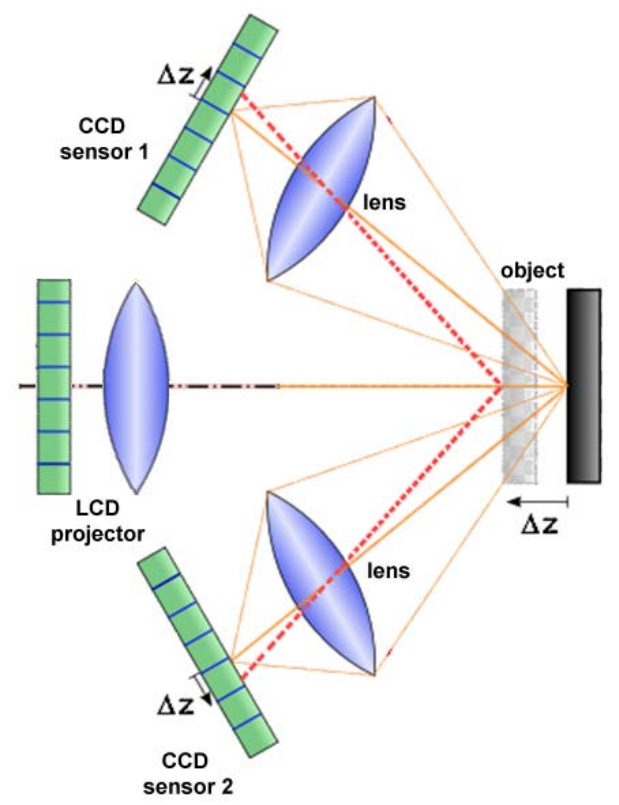

Fig. 1. Principle of optical triangulation by stereo vision used by SYMCAD ${ }^{\mathrm{TM}}$

The process of triangulation consists in determining the location of a 3D point of the object by measuring angles to it from known points (the projection centres of both video cameras). Depending on the distance at which each stripe projected by the video projector strikes the surface of the object from a reference point ( Z), the point of that stripe appears at different places in both camera fields of view ( $\left.z^{\prime}\right)$. A triangle is formed by the target dot on the object and the optical centres of both cameras. By looking at the location of the target dot in both camera fields of view, the location of the target dot is obtained.

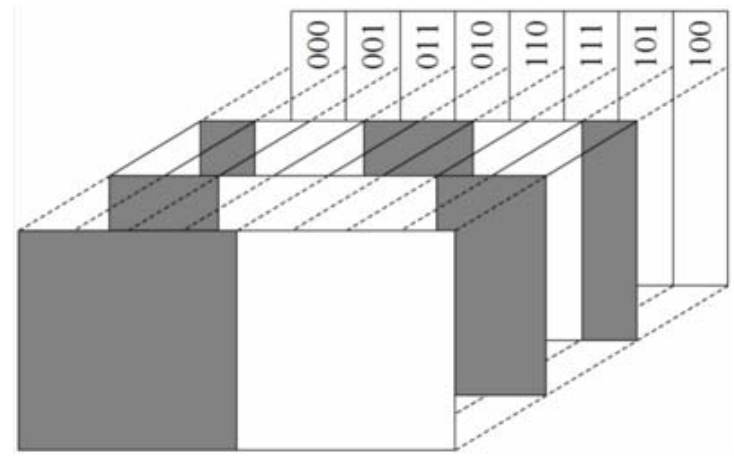

Fig. 2. Enhanced numeration of stripes through a special encoding of the structured light (Gray code)

By projection of a sequence of binary coded stripe patterns, the subsequent light levels for each pixel are forming a unique binary word revealing the individual stripe number. To minimize the effect of bit errors, the stripe patterns are designed to change only one bit per stripe.

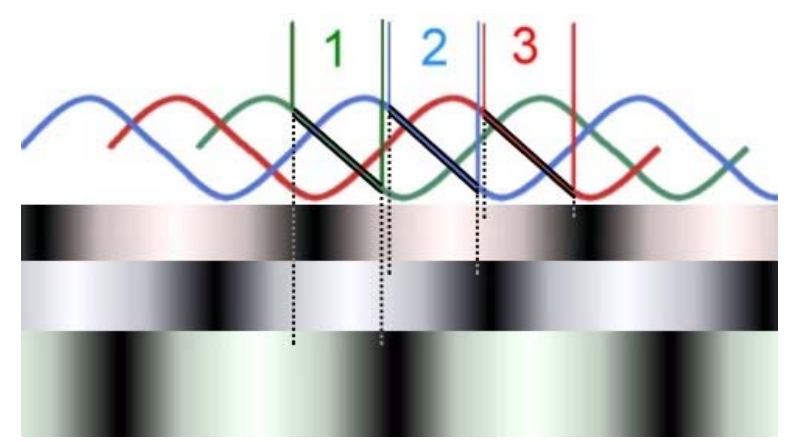

Fig. 3. Enhanced resolution through the projection of additional patterns (Phase Shifting method)

Three phase-shifted patterns ( /2, , 3 /2) are added to the reference pattern (full set of stripes) in order to enhance the resolution of the $3 D$ reconstructed dataset. 
On top of this ultimate technology, TELMAT has developed special application software in order to make the SYMCAD ${ }^{\mathrm{TM}}$ Tailor solution fulfil the requirements of tailor-made clothing shops - see figure 4. Furthermore, TELMAT integrated a direct link with 2D and 3D made-to-measure pattern design tools and notably to the intuitive Pattern Design and the fast and accurate 3D Draping software CLO 3D, developed by the South Korean company CLO Virtual Fashion Inc.
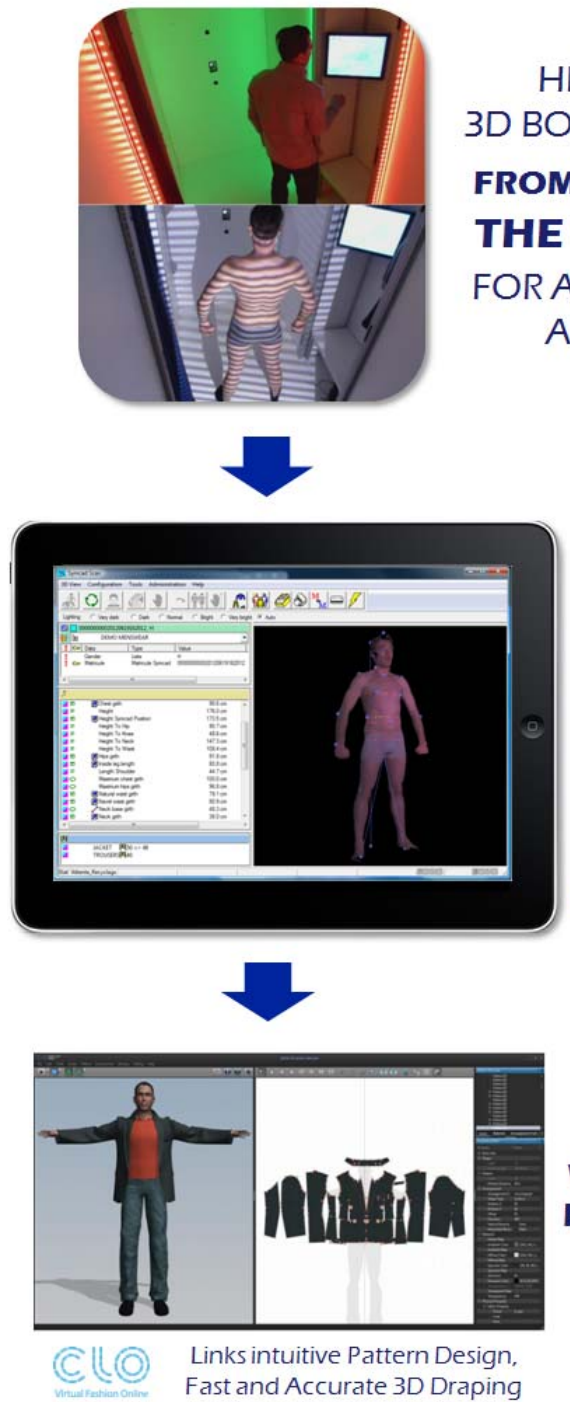

HIGH SPEED

3D BODY SCANNING

FROM 0.5 SECOND

THE FULL SCAN

FOR AN INCREASED

ACCURACY

\section{SOFTWARE \\ ADAPTED TO \\ MTM CLOTHING}

INTERACTIVE CONTROL

OF MEASURING POINTS

SEVERALMEASURING

SEQUENCES

BODY POSTURE ANALYSIS

\section{DIRECT LINK}

WITH 2D AND 3D

PATTERN DESIGN

TOOLS

Fig. 4. Architecture of the SYMCAD TM Tailor solution for tailor-made clothing shops.

The SYMCAD ${ }^{\mathrm{TM}}$ Tailor application software enables an interactive control of measuring points: anatomical landmarks are automatically detected by the measurement extraction algorithm but when the user moves such a measuring point, all body measurements related to this point are automatically modified. This feature accelerates the customization of tailor-made clothes according to the client's preferences.

Furthermore, the SYMCAD ${ }^{\text {TM }}$ Tailor application software enables several measuring sequences (with arms along the body and with arms apart) in order to take body measurements which are not affected by the client's posture imposed by the 3D body scanner. Four active landmarks may even be used by the operator to collect specific measuring points or to substitute existing anatomical landmarks of the system by the 3D locations of such active landmarks.

At last, a large set of body posture indicators are automatically collected to enable high-level body posture analyses which may be very useful for the tailoring process to take into account specific body postures. 


\subsection{Retail shops and online catalog shopping}

For retail shops and online catalog shopping, TELMAT has developed the special SYMCAD ${ }^{\text {TM }}$ Shop solution which may be based either on the same SYMCAD ${ }^{\mathrm{TM}}$ II technology or on the new low-cost SYMCAD ${ }^{\mathrm{TM}}$ Xt system using depth sensors (either ASUS Xtion ${ }^{\circledR}$ or MS Kinect ${ }^{\circledR}$ ) - see figure 5 . In this new version, the source of the projected structured light (white light) is replaced by a diffracted laser source (depth sensor).

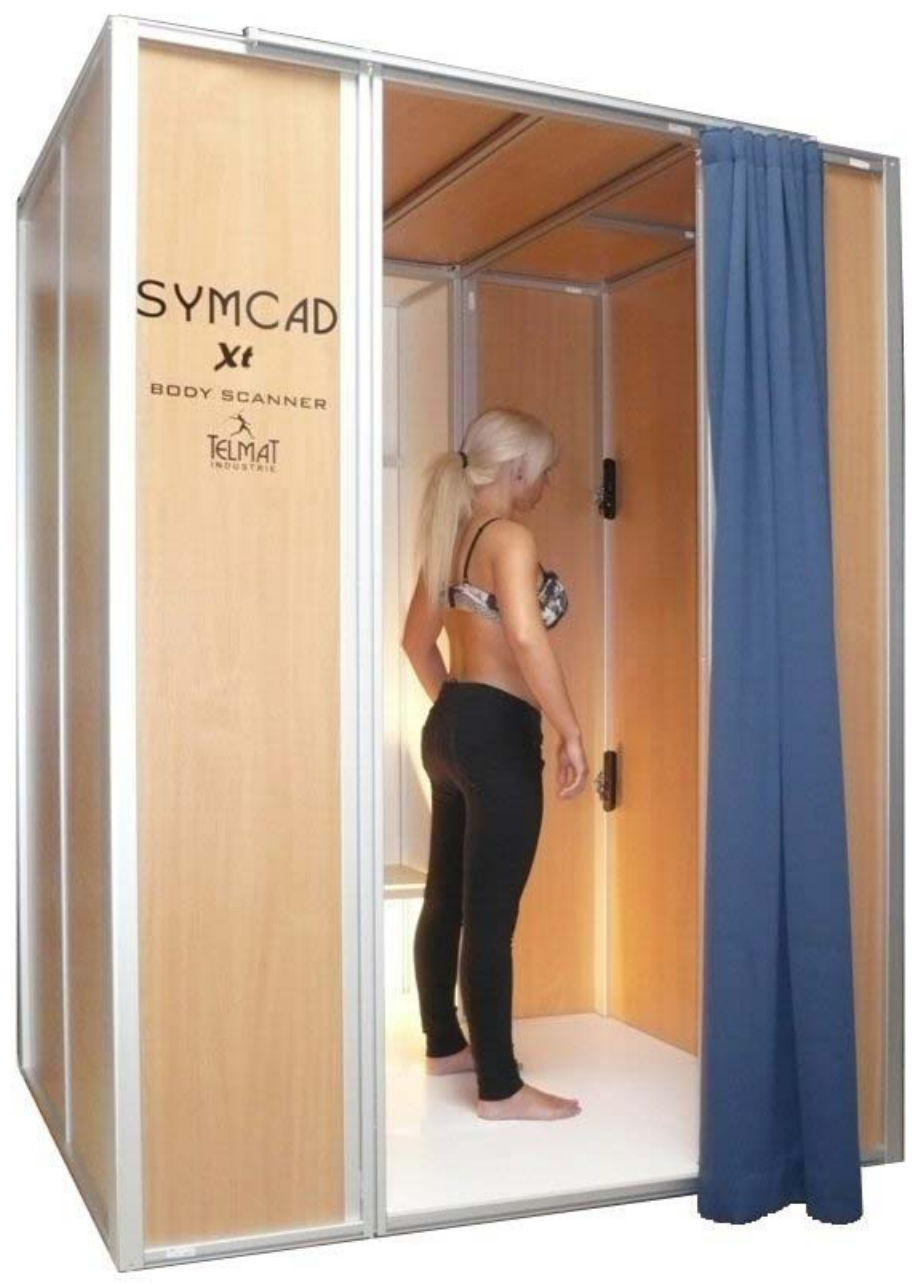

Fig. 5. SYMCAD ${ }^{\mathrm{TM}}$ Xt $3 D$ body scanner for retail shops, based on ASUS Xtion ${ }^{\circledR}$ depth sensors.

This SYMCAD ${ }^{\mathrm{TM}} \mathrm{Xt}$ system gathers the following benefits: low cost and reduced floor space which are very important for retailers of ready-to-wear products. However it cannot achieve neither the ultra fast time of scanning nor the automatic self-calibration process of SYMCAD ${ }^{\mathrm{TM}}$ II systems.

For retail shops and online catalog shopping, TELMAT has developed special application software in order to make the SYMCAD ${ }^{\mathrm{TM}}$ Shop solution fulfil the requirements of retailers - see figure 6 . Furthermore, TELMAT integrated a direct link with the fast and accurate 3D Draping software CLO 3D, developed by the South Korean company CLO Virtual Fashion Inc., which enables virtual try-on of 3D garments on the consumer's avatar, fitting analysis to show the stress map of the apparel fabric on the consumer's body, as well as virtual catwalks which can be used to promote new products by showing it worn by the consumer's avatar or by an avatar with an approaching morphotype. 


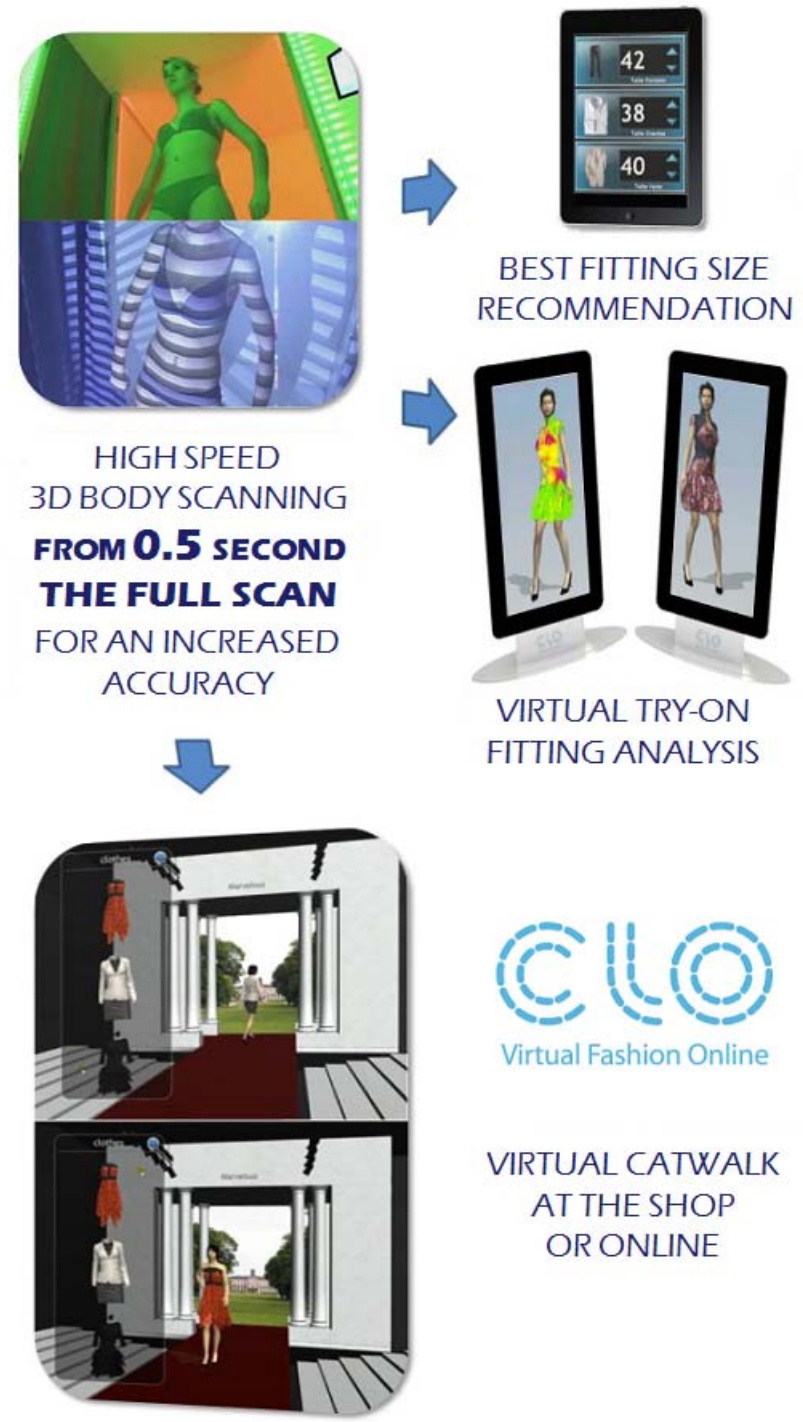

Fig. 6. Architecture of the SYMCAD ${ }^{\mathrm{TM}}$ Shop solution for retail shops and online catalog shopping.

\subsection{Body size measurement surveys}

All SYMCAD systems are also useful to undertake body size measurement surveys, either for apparel sizing improvement, in ergonomics (anthropometric studies), or for sport, health and fitness, where the client's body measurements must be surveyed - see figure 7 .

For this scope of applications, TELMAT has developed the special SYMCAD ${ }^{\text {TM }}$ Survey solution which may be based on the same SYMCAD ${ }^{\text {TM }}$ II technology or on the ultimate SYMCAD ${ }^{\text {TM }}$ ST system using up to 16 active landmarks to collect any specific body measurements required in the framework of such surveys.

The SYMCAD ${ }^{\text {TM }}$ Survey application software enables an interactive control of measuring points (ISO-8559 standard). It provides the user with several predefined measuring sequences according to the ISO-7250 standard (standing and sitting positions). New measuring sequences may also be defined by the user by using any combination of landmarks (standard or active landmarks traced by SYMCAD ${ }^{\mathrm{TM}}$ ST). SYMCAD ${ }^{\mathrm{TM}}$ ST automatically detects in 3D and identifies active landmarks set on the subject's body in order to calculate the appropriate anthropometric measures very quickly and efficiently.

Furthermore, it may integrate scanning peripherals (hand scanner, foot scanner and weighing machine). The SYMCAD ${ }^{\text {TM }}$ Survey application software merges all data coming from any measuring sequences or any scanning peripherals into a single database managed through a Relational Database Management System (R/DBMS). 

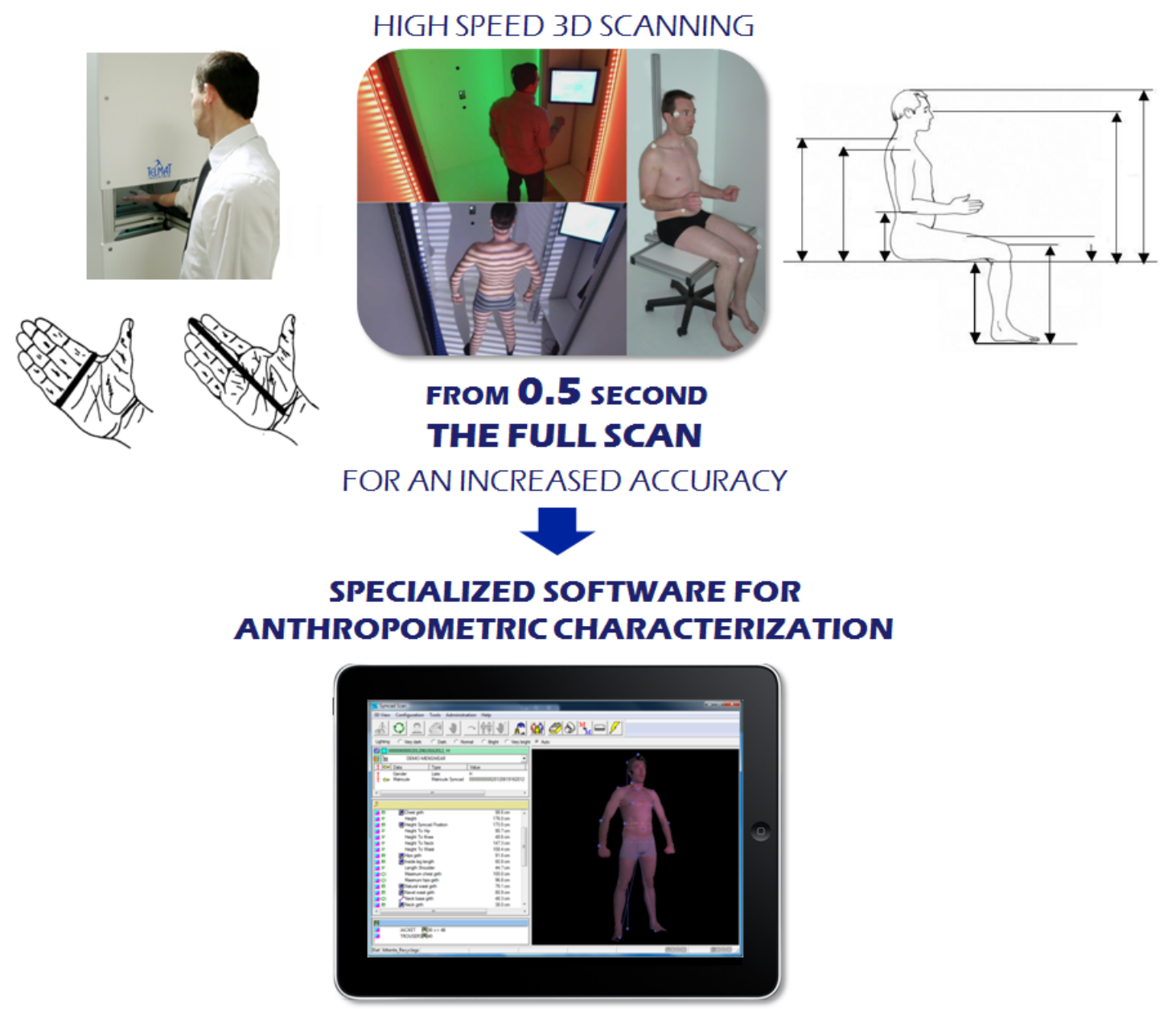

INTERACTIVE CONTROL OF MEASURING POINIS (ISO-8559 STANDARD) SEVERAL PREDEFINED MEASURING SEQUENCES (ISO-7250 STANDARD) AUTOMATC DEIECTION ANDIDENTIFICATON OF ACTIVE MARKERS

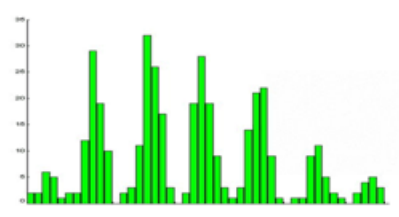

Fig. 7. Architecture of the SYMCAD TM Survey solution for body size measurement surveys.

\section{Perspectives: a wide range of products for a wide scope of applications}

TELMAT is extending its range of $3 D$ body scanning solutions named SYMCAD ${ }^{\text {TM }}$ to fulfill an even wider scope of applications in the apparel and fashion industry (tailor-made clothing shops, retail shops, online catalog shopping, body size measurement surveys) as well as in ergonomics (anthropometric studies), sport, health and fitness, entertainment, etc.

Very easy to use, SYMCAD ${ }^{\text {TM }}$ provides boutiques and clothing store chains with an automated body measurement system that can determine clothing sizes and help produce made-to-measure clothes.

SYMCAD ${ }^{\mathrm{TM}}$ II is a free-standing private booth and scanning system that is simple, autonomous and fun. Featuring automatic body positioning control, as well as a synthetic voice system that welcomes the customer into the booth, helps them find the ideal position, and then automatically triggers the measurement process. The person is inside a pleasant environment and a fun setting throughout. 
SYMCAD ${ }^{\mathrm{TM}}$ ST version (ST for Special Tracking) extends the range of applications of 3D body scanning to the domains of ergonomics, health and medicine. Specific scenarios of measurement extraction are defined to comply with all standardized predefined postures of the subject (sitting, standing or holding an object), according to the ISO-7250 standard. Once wireless active landmarks are set on the subject's body, SYMCAD ${ }^{\text {TM }}$ ST automatically detects and identifies them and calculates the appropriate anthropometric measures very quickly and efficiently.

Furthermore, SYMCAD ${ }^{\mathrm{TM}}$ enables to generate $3 \mathrm{D}$ avatars, either as $3 \mathrm{D}$ real data in VRML format, or 3D models warped from body measurement data for applications in virtual try-on or in entertainment.

Based on the ASUS Xtion ${ }^{\circledR}$ depth sensors, the new SYMCAD ${ }^{\mathrm{TM}}$ Xt system gathers new benefits: low cost and reduced floor space which are very important for retailers of ready-to-wear products. With the SYMCAD ${ }^{\mathrm{TM}}$ Shop application software, TELMAT has integrated a direct link with the fast and accurate 3D Draping software CLO 3D, developed by the South Korean company CLO Virtual Fashion Inc., which enables virtual try-on of 3D garments on the consumer's avatar, fitting analysis to show the stress map of the apparel fabric on the consumer's body, as well as virtual catwalks which can be used to promote new products by showing it worn by the consumer's avatar or by an avatar with an approaching morphotype. 